\title{
Differences in E-Cadherin and Syndecan-1 Expression in Different Types of Ameloblastomas
}

\author{
Ramón G. Carreón-Burciaga $\left(\mathbb{D},{ }^{1}\right.$ Rogelio González-González $\mathbb{D}^{1},{ }^{1}$ Nelly Molina-Frechero $\left(\mathbb{D},{ }^{2}\right.$ \\ Sandra López-Verdín, ${ }^{3}$ Vanesa Pereira-Prado, ${ }^{4}$ and Ronell Bologna-Molina ${ }^{4}{ }^{4}$
}

${ }^{1}$ Department of Research, School of Dentistry, Juarez University of the State of Durango, Durango, DGO, Mexico

${ }^{2}$ Department of Health Care, Xochimilco Unit, Autonomous Metropolitan University, Mexico City, Mexico

${ }^{3}$ Research Institute of Dentistry, Health Science Center, Guadalajara University, Guadalajara, JAL, Mexico

${ }^{4}$ Molecular Pathology Area, School of Dentistry, University of the Republic, Montevideo, Uruguay

Correspondence should be addressed to Rogelio González-González; rogegg@hotmail.com

Received 21 December 2017; Revised 8 March 2018; Accepted 14 March 2018; Published 23 April 2018

Academic Editor: Consuelo Amantini

Copyright (c) 2018 Ramón G. Carreón-Burciaga et al. This is an open access article distributed under the Creative Commons Attribution License, which permits unrestricted use, distribution, and reproduction in any medium, provided the original work is properly cited.

\begin{abstract}
Ameloblastomas are a group of benign, locally aggressive, recurrent tumors characterized by their slow and infiltrative growth. ECadherin and syndecan-1 are cell adhesion molecules related to the behavior of various tumors, including ameloblastomas. Ninetynine ameloblastoma samples were studied; the expression of E-cadherin and syndecan-1 were evaluated by immunohistochemistry. E-Cadherin and epithelial syndecan-1 were more highly expressed in intraluminal/luminal unicystic ameloblastoma than in mural unicystic ameloblastoma and solid/multicystic ameloblastoma, whereas the stromal expression of syndecan-1 was higher in mural unicystic ameloblastoma and solid/multicystic ameloblastoma. Synchronicity was observed between E-cadherin and epithelial syndecan-1; the expression was correlated with intensity in all cases. There was a strong association between expression and tumor size and recurrence. The evaluation of the expression of E-cadherin and syndecan-1 are important for determining the potential aggressiveness of ameloblastoma variants. Future studies are required to understand how the expression of these markers is related to tumor aggressiveness.
\end{abstract}

\section{Introduction}

The ameloblastoma is an epithelial neoplasia originating in the enamel and has been described as a tumor that is usually unicentric, intermittent in growth, and persistent [1]. According to the recent classification by the World Health Organization (WHO) [2], ameloblastoma is defined as a benign epithelial odontogenic neoplasia, characterized by tumor expansion, progressive growth, and a tendency for recurrence if not completely removed. Ameloblastomas are classified as follows according to their clinical and histopathological features: solid multicystic (SMA), unicystic (UAM), extraosseous/peripheral, and metastasizing ameloblastoma; the two most common types are SMA and UAM [2]. SMA is an aggressive tumor with high recurrence rates if not treated promptly; the early manifestation of this tumor is characterized by slow growth and painless expansion, which later exhibits accelerated growth with several complications that can be fatal if its growth is not controlled. SMA has two common types of histopathological growth patterns that are not related to prognosis [2]. UAM is characterized by slow growth that occurs as a single cystic cavity, in which different types of epithelial extension can occur, namely, luminal, intraluminal (UAM-L/I), and mural (UAM-M). The mural component displays aggressive behavior, like that observed in SMA [2].

The loss of cellular adhesion plays an important role in the invasion and growth of tumor cells which are among the first events that occur in tumors of epithelial origin, such as ameloblastomas [3]. E-Cadherin (Ecad) is a calciumdependent cell adhesion molecule that is expressed in epithelial tumors and is associated with prognosis; reduced or 
eliminated Ecad expression is associated with progression, invasion, and a poor prognosis in these types of tumors [4-7]. Syndecan-1 is a transmembrane proteoglycan that is expressed in fibroblasts and epithelial cells; it plays an important role in numerous biological process, such as cytoskeleton organization, cell-cell adhesion, and cell-extracellular matrix (ECM) adhesion. Syndecan-1 (Syn1) mediates interactions with ECM molecules through its heparan sulfate chains and interacts with heparin-binding growth factors, cytokines, proteinases, and proteinase inhibitors. It is considered an important structural maintenance protein $[4,8]$. Syn 1 is mainly localized in the basolateral surface of epithelial cells and occasionally in the stroma of mature epithelial cells [4]. The epithelial expression of Syn1 (Syn1E) has been studied in several tumors; its expression, which can range from overexpression to complete absence, has been related to tumor behavior [9-12]. The stromal expression of Syn1 (Syn1S) is related to alterations in fibronectin and ECM organization; additionally, its expression is associated with angiogenesis and it enhances the proliferation of endothelial cells and promotes the proliferation of tumor cells [13]. In ameloblastomas, the reduction or termination of expression of Syn1E is related to tumor progression and invasion, while Syn1S expression is related to cell invasion, tumor progression, and metastasis.

The expression profiles of Ecad, Syn1E, and Syn1S have been evaluated separately in previous studies [12, 14-19]. However, there is minimal research on the comparisons between the expressions of Ecad, Syn1E, and Syn1S in the same system, in which a greater understanding of the behavior of these tumors can be obtained by analyzing their expression profiles.

\section{Materials and Methods}

2.1. Patients. A total of ninety-nine patients treated for ameloblastoma were analyzed; clinical data and blocks of paraffin were obtained from the Oral Pathology Services of the School of Dentistry of the University of the Republic, Uruguay. Data regarding age, gender, tumor localization, tumor size, radiographical parameters, and histopathological parameters were evaluated. The histopathological diagnoses were reevaluated by two pathologists with experience in odontogenic tumors according to the latest classifications of the WHO [2]. Of the cases evaluated, there were thirty-eight cases of both UAM-L/I and SMA and twenty-three cases of UAM-M.

2.2. Immunohistochemistry. For immunohistochemical analyses, $3 \mu \mathrm{m}$ thick sections were prepared and mounted on slides treated with poly-L-lysine. The sections were deparaffinized in an oven at $60^{\circ} \mathrm{C}$ for $30 \mathrm{~min}$ and then incubated in xylol for $5 \mathrm{~min}$. The sections were hydrated in a graded alcohol series (100, 90, 70, and 50\%) and washed with distilled water. To unmask the epitopes, antigen retrieval was performed with $10 \mathrm{mM}$ sodium citrate solution, with a high or low $\mathrm{pH}$ depending on the characteristics of each antibody. This recovery technique was performed in a microwave pressure pot with a maximum power of $750 \mathrm{~W}$ for two $5 \mathrm{~min}$ cycles, allowed to cool to room temperature, and rinsed with distilled water. Endogenous peroxidases were blocked with $0.9 \%$ hydrogen peroxide, followed by rinsing in distilled water and phosphate-buffered saline (PBS), $\mathrm{pH}$ 7.4. Samples were incubated for $45 \mathrm{~min}$ with primary antibodies against Ecad (Clone NCH-38, 1:100, monoclonal mouse anti-human, Dako, Santa Clara, CA, USA) and Syn1 (CD-138, Clone MIB 15, 1:100, monoclonal mouse anti-human, Dako). Subsequently, sections were incubated with a second biotinylated anti-mouse/anti-rabbit antibody and streptavidin/peroxidase complex (LSA-B + labeled stre ptavidin-biotin, Dako) for $30 \mathrm{~min}$ each. The products were visualized using 3,3'-diaminobenzidine- $\mathrm{H}_{2} \mathrm{O}_{2}$ (Dako). Fragments of epithelial tissue were used as positive controls, and incubation with the primary antibodies was omitted for negative controls.

2.3. Rate of Expression. Ecad and Syn1E can be observed in the cell membrane and cytoplasm of cells. The expressions of Ecad and Syn1E were calculated by light microscopy, and photomicrographs were obtained using a digital camera (Olympus C-7070) in four fields where neoplastic cells were more abundant along cystic lines and wall follicles. An average was then obtained based on the sum of the percentages of each of the fields for each case evaluated. Syn1S expression was analyzed in tumor-stromal cells and inflammatory cells, using the same technique as that for Ecad and Syn1E for the evaluation of stromal expression, without considering the expression of inflammatory cells.

2.4. Intensity of Expression. In the same photomicrographs, the expression intensities of Ecad and Syn1E were also evaluated in the cell membrane using the Image ImmunoMembrane plug-in (BioMediTech, Tampere, Finland). The technique was performed as reported previously by Tuominen et al. [20]. The intensity was scored as negative (0) when the intensity of membrane expression in the neoplastic cells was negative or $\leq 10 \%$ (Figure $1(\mathrm{a})$ ), and it was scored as positive when the percentage was $>10 \%$ according to whether the intensity was weakly positive (1 + ), moderately positive $(2+)$, or strongly positive $(3+)$ (Figures 1(b)-1(d), resp.). Figures 1(e) and 1(f) show the positive controls for expression of Ecad and Syn1, respectively. Owing to the stromal expression characteristics of Syn1S, its expression intensity could not be evaluated.

2.5. Statistical Analysis. Descriptive analysis was performed to describe the clinical features. Spearman's rank correlation coefficient $(\rho)$ was used to evaluate the relationship between the rate and intensity of membrane expression. The KruskalWallis test was used to determine the differences in rates of expression and clinical features. The Mann-Whitney $U$ test was conducted to determine the differences in rates of expression between two groups. The results were considered statistically significant at $p \leq 0.05$, and the rank correlation coefficient $(\rho)$ was categorized as moderately positive $(\rho=$ $0.50-0.70)$, highly positive $(\rho=0.70-0.90)$, and very highly positive $(\rho=0.90-1.00)$. 


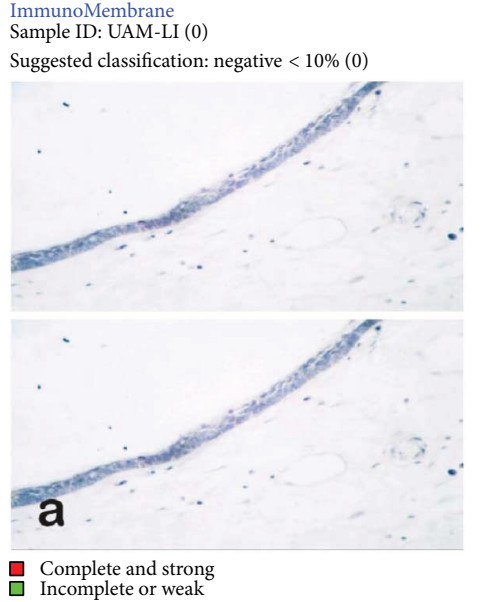

(a)

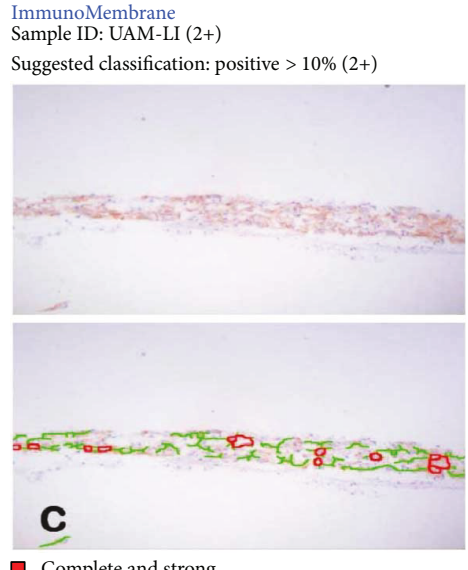

Complete and strong
Incomplete or weak

(c)

ImmunoMembrane

Sample ID: E-cadherin control

Suggested classification: positive $>10 \%(3+)$
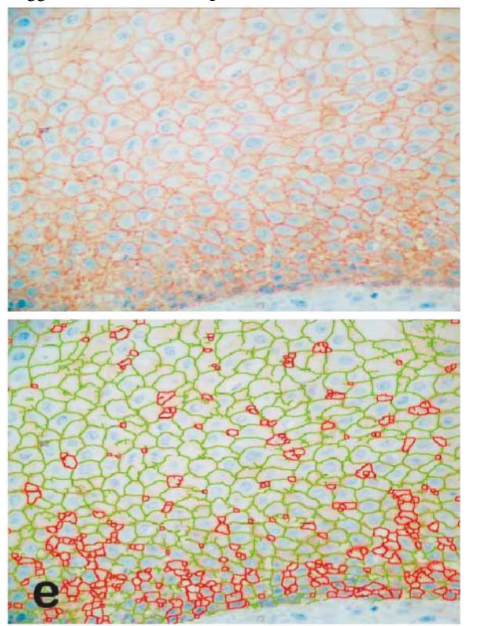

Complete and strong Incomplete or weak

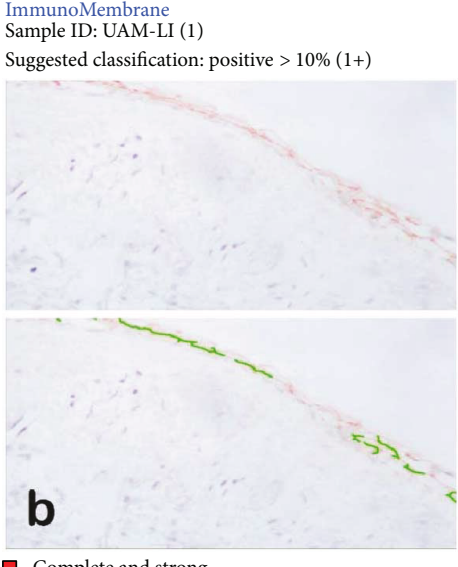

Complete and strong
Incomplete or weak

(b)

ImmunoMembrane

Sample ID: UAM-LI (3+)

Suggested classification: positive $>10 \%(3+)$
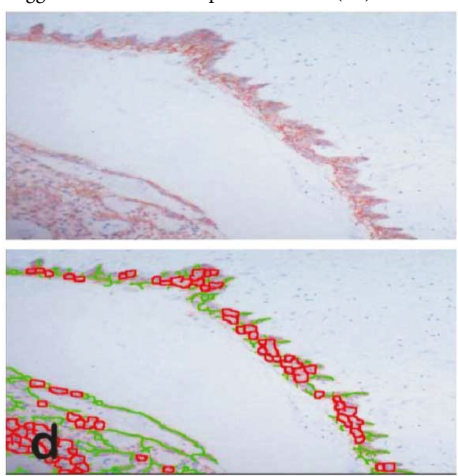

Complete and stron

Incomplete or weak

(d)

mmunoMembrane

Sample ID: syndecan-1 control

Suggested classification: positive $>10 \%(3+)$
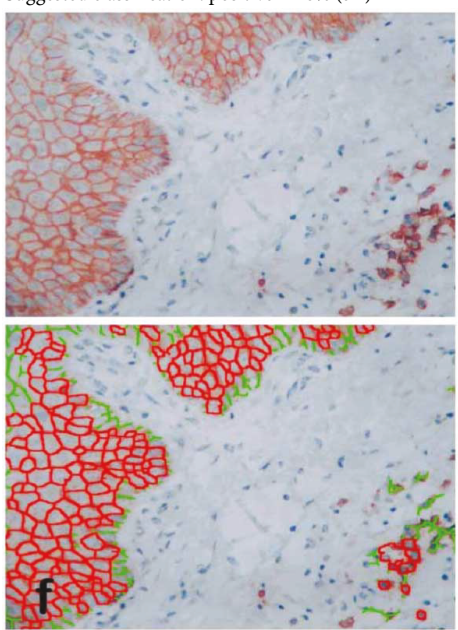

Complete and stron
Incomplete or weak

(e)

(f)

FIgURE 1: Intraluminal/luminal unicystic ameloblastomas in which the expression of E-cadherin was evaluated using ImmunoMembrane software. (a) Negative (or $<10 \%)$ immunostaining, intensity (0); (b) positive $(\geq 10 \%)$ immunostaining, intensity (1+); (c) positive immunostaining, intensity (2+); (d) positive immunostaining, intensity (3+); (e) group control for E-cadherin expression in oral mucosa, positive immunostaining, intensity (3+); (f) group control for syndecan-1 expression in oral mucosa, positive immunostaining, intensity (3+). 


\section{Results}

3.1. Clinical Features and Rates of Expression. UAM-L/I and UAM-M were more frequently observed in male patients, at a ratio of $1.2: 1$ and $2.3: 1$, respectively; in contrast, the gender ratio of SMA cases was $1: 1$. With respect to age, all types were most frequently observed in patients aged $<30.4$ years. Thirty-four tumors were found in the posterior region of the mandible, followed by the anterior region of the mandible with a total of three cases. The unilocular radiographic pattern was the most frequent-with a total of fifty-four cases-mostly in UAM-L/I tumors, while the multilocular pattern was more frequent in SMA tumors. A total of sixteen cases were classified as unknown cases, as they were not registered in the medical records at the time of this study. A size of $<5.3 \mathrm{~cm}$ was the most frequent, appearing at the same frequency in UAM-L/I and SMA tumors; tumors sized $>5.3 \mathrm{~cm}$ showed a similar distribution. Only seven cases were recurring and were identified more often in cases of SMA (Table 1).

The correlation between the expression of Ecad, Syn1E, and Syn1S and tumor size and recurrence is presented in Table 2 .

\subsection{Expression Localization}

3.2.1. UAM-L/I. Ecad and Syn1E were expressed in the cytoplasm of tumor cells in the neoplastic cystic epithelium (luminal), in the intraluminal neoplastic cells, and in cells similar to the stellate reticulum; the highest expression was observed in neoplastic basal cells and the stellate reticulum (Figures 2(a) and 2(b)). Syn1S was present in the tumor stroma surrounding the neoplastic cells (Figure 2(b)).

3.2.2. UAM-M. Ecad and Syn1E were present in the cytoplasm of the neoplastic cystic epithelium, in the intraluminal projections, and in tumor islands contained within the cystic wall. The highest rates of expression were observed in neoplastic basal cells and in the stellate reticulum (Figures 2(c) and $2(\mathrm{~d}))$. Syn $1 \mathrm{~S}$ was observed in the connective tissue adjacent to both the neoplastic cystic epithelium and the neoplastic epithelial islands contained within the cystic wall (Figure 2(d)).

3.2.3. SMA. Ecad and Syn1E were present in the neoplastic islands of the follicular pattern and in the cords of the neoplastic epithelium of the plexiform pattern, predominating in the tumor basal cells and in the cells resembling the stellate reticulum (Figures 2(e) and 2(f)). Syn1S was observed in the connective tissue close to the islands and cords of epithelial neoplastic cells (Figure 2(f)).

3.3. Expression Intensity. Ecad and Syn1E showed similar expression levels ( $<1 \%$ difference); they were expressed at higher levels in UAM-L/I. Furthermore, their expression levels were significantly different only in UAM-M samples (Table 3). Syn1S expression was higher in SMA and UAM$M$ and differed by $<5 \%$ between the two variants, while the lowest expression was observed in UAM-L/I samples (Table 3). The expression intensity of Ecad and Syn1E was
TABle 1: Distribution of ameloblastomas according to clinical features.

\begin{tabular}{|c|c|c|c|}
\hline Clinical features & $\begin{array}{l}\text { UAM-L/I } \\
(n=38)\end{array}$ & $\begin{array}{l}\text { UAM-M } \\
(n=23)\end{array}$ & $\begin{array}{c}\text { SMA } \\
(n=38)\end{array}$ \\
\hline \multicolumn{4}{|l|}{ Gender } \\
\hline Male & $21(55.3 \%)$ & $16(69.6 \%)$ & $19(50 \%)$ \\
\hline Female & $17(44.7 \%)$ & $7(30.4 \%)$ & $19(50 \%)$ \\
\hline \multicolumn{4}{|l|}{ Mean age, years } \\
\hline$<30.4$ & $26(68.4 \%)$ & $14(60.9 \%)$ & $17(44.7 \%)$ \\
\hline$\geq 30.4$ & $12(31.6 \%)$ & $9(39.1 \%)$ & $21(55.3 \%)$ \\
\hline \multicolumn{4}{|l|}{ Site of localization } \\
\hline \multicolumn{4}{|l|}{ Maxilla } \\
\hline Anterior & $2(5.3 \%)$ & 0 & 0 \\
\hline Posterior & $1(2.6 \%)$ & 0 & 0 \\
\hline \multicolumn{4}{|l|}{ Mandible } \\
\hline Anterior & $1(2.6 \%)$ & $1(4.3 \%)$ & $1(2.6 \%)$ \\
\hline${ }^{*}$ Posterior & $34(89.4 \%)$ & $22(95.7 \%)$ & 37 (97.4\%) \\
\hline \multicolumn{4}{|l|}{ Radiographic } \\
\hline Unilocular & $26(68.4 \%)$ & $16(69.6 \%)$ & $12(31.6 \%)$ \\
\hline Multilocular & $8(21 \%)$ & $3(13 \%)$ & $18(47.4 \%)$ \\
\hline Unknown & $4(10.5 \%)$ & $4(17.4 \%)$ & $8(21 \%)$ \\
\hline \multicolumn{4}{|l|}{ Size $(\mathrm{cm})$} \\
\hline$<5.3$ & $23(60.5 \%)$ & $17(74 \%)$ & $23(60.5 \%)$ \\
\hline$\geq 5.3$ & $15(39.4 \%)$ & $6(26 \%)$ & $15(39.5 \%)$ \\
\hline \multicolumn{4}{|l|}{ Recurrence } \\
\hline Yes & $2(5.3 \%)$ & $2(8.7 \%)$ & $3(7.9 \%)$ \\
\hline No & $36(94.7 \%)$ & $21(91.3 \%)$ & $35(92.1 \%)$ \\
\hline
\end{tabular}

${ }^{*}$ Body and/or ramus.

$2+$ in all cases, with a moderate to high positive correlation coefficient (Figure 3, Table 4).

\section{Discussion}

Ameloblastomas are locally aggressive, recurrent, benign tumors with the potential for malignant transformation into ameloblastic carcinomas [18]. UAM and SMA are most often located in the ascending limb of the mandible, followed by the body and the mandibular symphysis. Radiographically, they present as well-defined unilocular neoplasms and, in most cases, are associated with impacted dental organs, sometimes showing root resorption and cortical perforation [2]. Recent WHO guidelines [2] indicate that invasion of the cystic wall (mural invasion) by ameloblastic tumor cells in UAM is an important histopathological factor, as this mimics a behavior similar to that found in SMA [2].

In general, there are several molecular processes involved in ameloblastoma tumor progression [21]. These include proteins related to cell adhesion, apoptosis regulation, cell cycle, cell proliferation, BRAF V600 mutations, tumor front, angiogenesis, bone remodeling, and the ECM [22-29].

Cell adhesion molecules are altered in various neoplasms, especially malignant ones, favoring progression, 


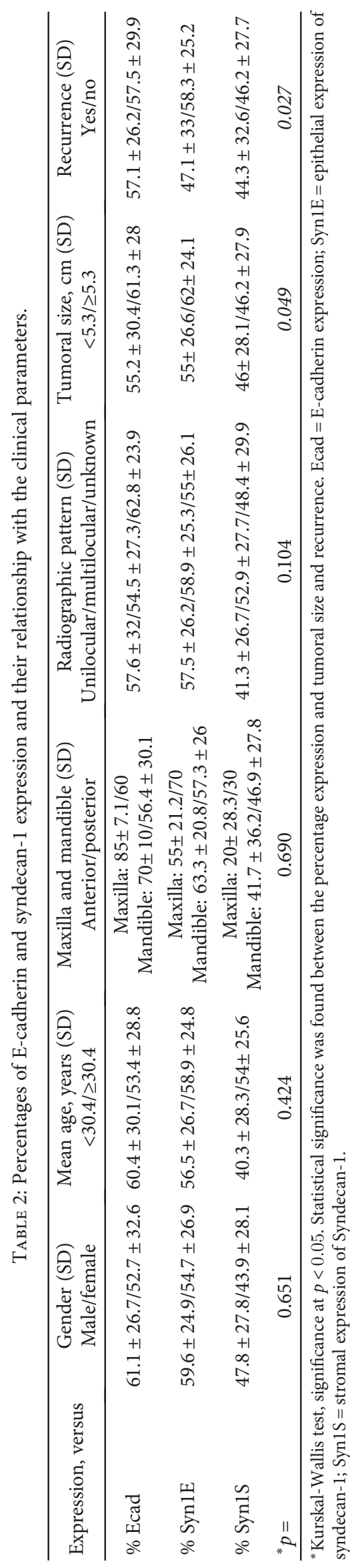




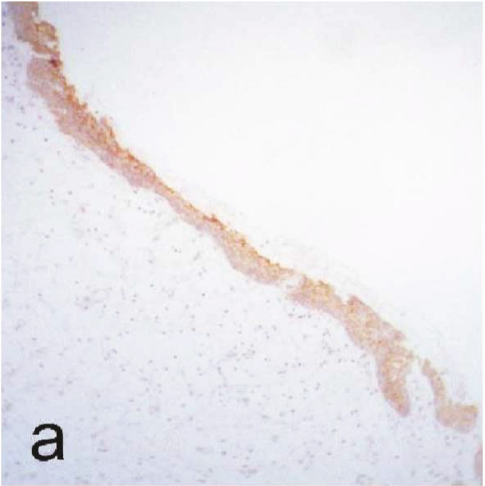

(a)

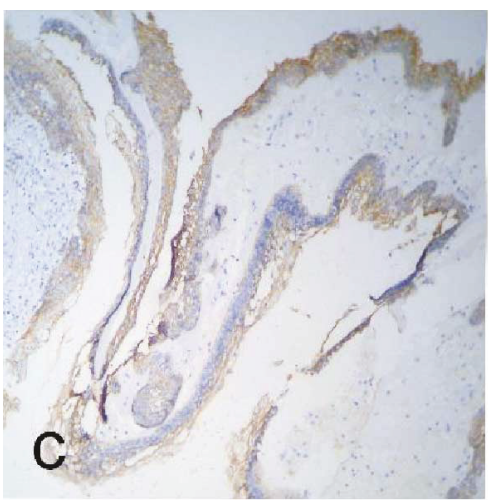

(c)

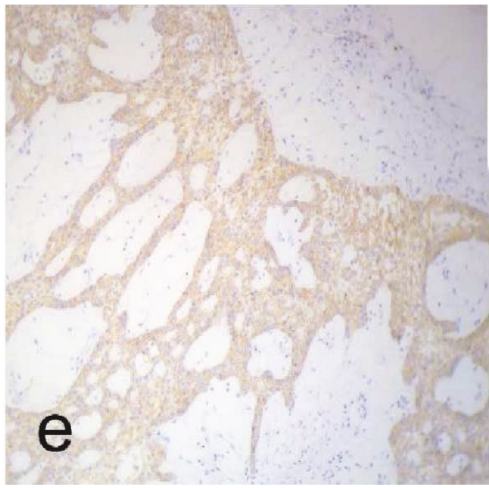

(e)

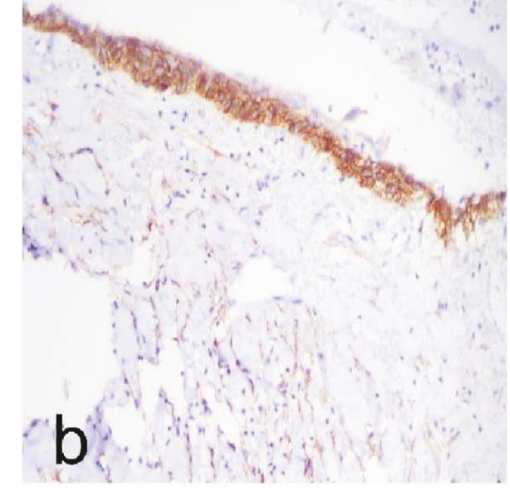

(b)

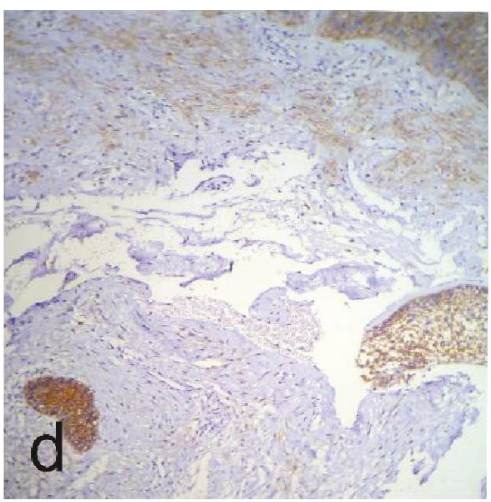

(d)

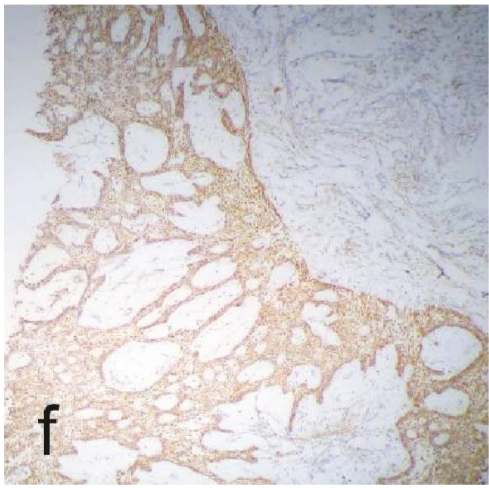

(f)

Figure 2: Ameloblastomas in which the rates of E-cadherin and syndecan-1 expression were evaluated. (a) E-Cadherin expression in a luminal/intraluminal ameloblastoma. (b) Expression of epithelial and stromal syndecan-1 in a luminal/intraluminal ameloblastoma. (c) Expression of E-cadherin in a unicystic ameloblastoma with mural invasion. (f) Membrane expression intensity of E-cadherin in a unicystic ameloblastoma with mural invasion. (d) Expression of epithelial and stromal syndecan-1 in a unicystic ameloblastoma with mural invasion. (e) Expression of E-cadherin in a solid/multicystic ameloblastoma. (f) Epithelial and stromal expression of syndecan-1 in a solid/multicystic ameloblastoma.

invasion, recurrence, and metastasis $[3,30]$. Ecad is a molecule with tumor suppressor functions; it is possible that these functions are related to the capacity of cells to adhere because in normal cells, growth and migration are inhibited when one cell adheres to another [30]. The loss of Ecad in most tumors of epithelial origin may be associated with aggressiveness, tumor invasion, and metastasis $[4,30,31]$. Our results show that the rate of Ecad expression was the highest in UAM-L/I tumors. Ecad expression was highly similar between UAM-L/I and UAM-M samples, suggesting that Ecad expression may be related to a decrease in cell adhesion capacity and, therefore, to the potential for the tumor cell to invade the cystic wall. This possibility could be supported by the results obtained for SMA, whose expression profiles were lower than those for UAM-L/I and UAM-M.

Martinez-Martinez et al. [19] reported that the expression levels of Ecad and B-catenin were low in ameloblastomas 
TABLE 3: Differences in expression among UAM-L/I, UAM-M, SMA, and E-cadherin and syndecan-1 expression.

\begin{tabular}{lccc}
\hline Ameloblastoma & $\begin{array}{c}\text { \% Ecad versus values } \\
\text { \% Syn1E }\end{array}$ & $\begin{array}{c}\text { \% Ecad versus } \\
\text { \% Syn1S }\end{array}$ & $\begin{array}{c}\text { \% Syn1E versus } \\
\text { \% Syn1S }\end{array}$ \\
\hline UAM-L/I & 0.088 & 0.001 & 0.001 \\
UAM-M & 0.010 & 0.012 & 0.010 \\
SMA & 0.881 & 0.180 & 0.123 \\
\hline
\end{tabular}

${ }^{*}$ Mann-Whitney $U$ test, statistical significance at $p<0.05$. Ecad $=$ E-cadherin expression; Syn1E = epithelial expression of syndecan-1; Syn1S = stromal expression of syndecan-1.

and ameloblastic carcinomas, consistent with our results. This suggests that Ecad is associated with tumor behavior due to the loss of cell adhesion. Ecad expression in cells resembling the stellate reticulum was observed in the three tumor variants included in this study, which could demonstrate, as shown by Pereira et al. [17], that increased expression of Ecad promotes adhesion between distant cells, as observed in UAM-L/I tumors.

Syndecans are a family of heparan sulfate proteoglycans that are expressed on the surface of adherent cells and in many nonadherent cells. Syndecans consist of a family of four members; Syn 1 is the most important and most well-characterized. Syn 1 plays a major role in cell-cell and ECM adhesion, interacts with various heparin-binding growth factors, and is an important regulator of cell adhesion and ECM molecules [16, 24, 32]. In tumor biology, Syn1 has been reported as a prognostic indicator for several malignancies, as its expression levels have a strong association with tumor invasion, proliferation, and cellular adhesion [12]. In head and neck carcinomas, decreased or absent Syn1 expression is associated with aggressiveness and a poor prognosis $[14,25]$.

The results of our study showed that the rate of Syn1E expression was higher in UAM-L/I, consistent with those of previous studies [12]. Interestingly, Ecad and Syn1E had a difference in expression of $<1 \%$; therefore, as in previous studies [26], it is possible that the minimal difference in Ecad and Syn1E expression in ameloblastomas is related to the coordination and regulation of expression between these two proteins and that the synchronous increase or decrease in expressed is associated with the adhesive capacity of tumor cells. Several studies have associated the synchronous reduction of Ecad and Syn1E with the processes of carcinogenesis and used it as an indicator of prognosis [26]. Syn1S expression is related to the regulation of cell growth, cell proliferation, the capacity to interact with heparin-binding growth factor family members, induction of angiogenesis, growth promotion, and tumor progression; it contributes to the invasion and metastasis of malignant tumors $[14,25]$.

Our data suggest that UAM-L/I tumors have cohesive cell groups that possess a lower invasive capacity and result in a better prognosis, unlike UAM-M tumors, which display less cohesive cell groups that possess a more invasive phenotype, resulting in bone reabsorption, cystic growth, and subsequent mural invasion. SMA tumors are more aggressive than UAM tumors [2]; our results show that UAM-L/I exhibited a higher expression of Ecad and Syn1E, but not Syn1S, which was expressed at lower levels than in SMA. It is interesting to note that UAM-M tumors were found to be highly similar to SMA. In our study, a low difference in Syn1S expression was observed between UAM-M and SMA tumors, which could indicate that the observed expression patterns are related to the similar behaviors of the UAM-M and SMA tumors; conversely, UAM-L/I tumors display less aggressive behavior, consistent with the recent WHO guidelines, which noted that UAM with mural invasion displays behaviors similar to SMA [2].

We did not find any associations between clinical characteristics such as gender, age, localization, and radiographical patterns; however, we found that the expression of Syn1S was somewhat higher in males, patients aged $<30.4$ years, the posterior region of the mandible, and multilocular radiographic patterns, suggesting that these data may be associated with tumor aggressiveness. The radiographic characteristics seem to be relevant for the prognosis of ameloblastoma, as the multilocular radiographic pattern was found to be related to a higher incidence of recurrence [33]. In our study, the multilocular radiographic pattern was more frequently associated with SMA, and this variant was more frequently associated with recurrence. Therefore, the multilocular radiographic pattern is an important risk factor that should be considered at the time of surgical treatment, as it is possible that the multilocular pattern is associated with cell invasion.

We found that the expression of Ecad and Syn1E in recurrent ameloblastomas was minimal compared to that in nonrecurrent ameloblastomas; although the difference in expression was not high, it was significant, especially between Syn1E and Syn1S. This result could indicate that tumor invasion is related to alterations in cell-cell and cell-ECM interactions, mediated by cell adhesion molecules [34]; it is possible that the gradual loss of the expression of Ecad and Syn1E as well as the gradual increase in Syn1S expression are related to the promotion of tumor cell invasion to the stroma, and with the capacity to gain migratory capability and autonomous cell survival. This hypothesis is supported by our results, which showed a higher expression of Ecad, Syn1E, and Syn1S in ameloblastomas $\geq 5.3 \mathrm{~cm}$ in size compared to those $<5.3 \mathrm{~cm}$ in size. This could indicate that the growth of ameloblastomas is slow and constant and that during growth, these cells acquire a migratory and invasive capacity, forming tumor nests related to recurrence.

\section{Conclusion}

In this study, we evaluated clinical, radiographic, and histopathological findings from ninety-nine cases of ameloblastoma and related these data to the expression of Ecad, Syn1E, and Syn1S. Importantly, we found that the expression of these proteins had an association with radiographic patterns and tumor size as important risk factors for recurrence. We established a possible relationship between Ecad 


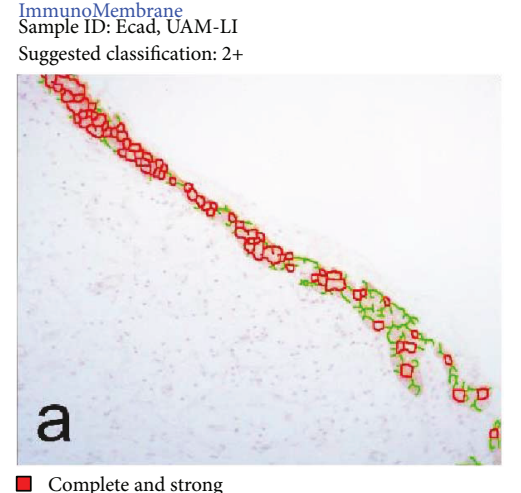

Complete and strong
Incomplete or weak

(a)

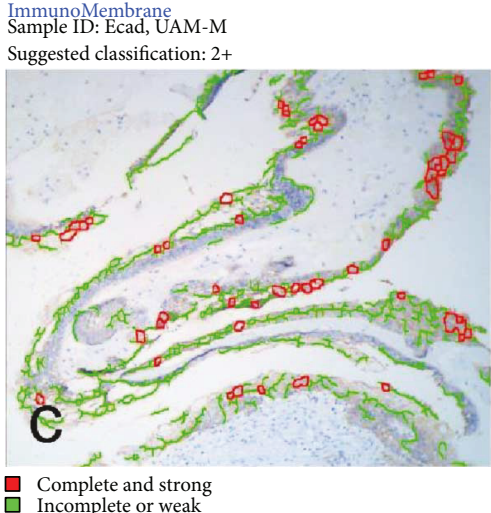

(c)

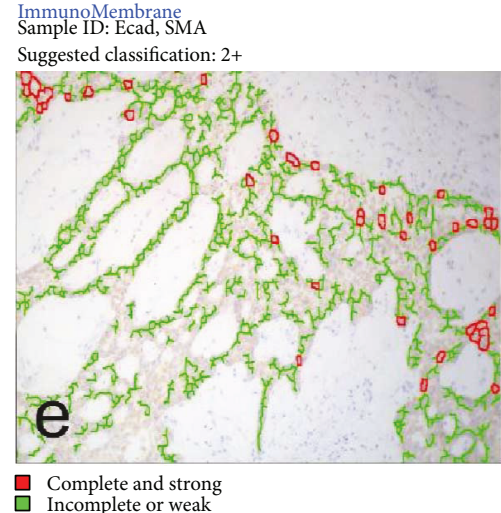

(e)

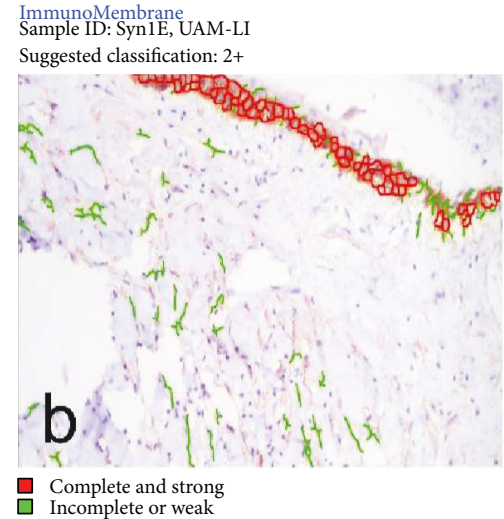

(b)

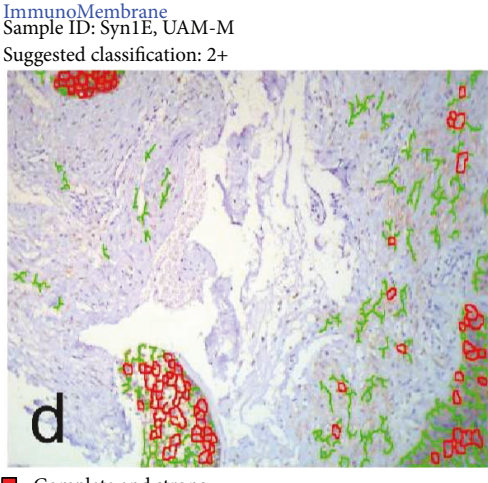

Complete and strong

(d)

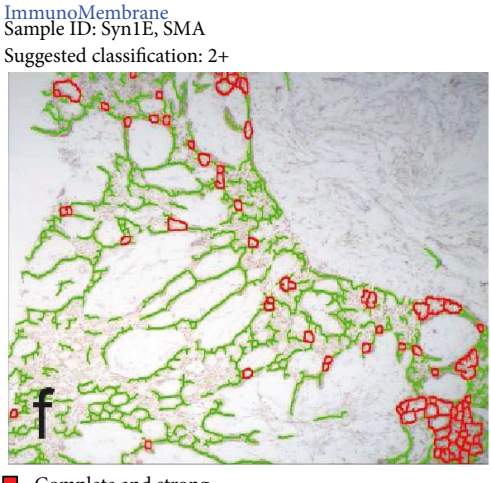

Complete and strong
Incomplete or weak

(f)

Figure 3: Ameloblastomas in which the membrane expression of E-cadherin and syndecan-1 were evaluated. (a) Membrane expression of E-cadherin in a luminal/intraluminal ameloblastoma. (b) Membrane intensity expression of epithelial syndecan-1 in an intraluminal/ luminal ameloblastoma. (c) Membrane intensity expression of E-cadherin in a unicystic ameloblastoma with mural invasion. (d) Membrane intensity expression of epithelial syndecan-1 in a unicystic ameloblastoma with mural invasion. (e) Membrane intensity expression of E-cadherin in a solid/multicystic ameloblastoma. (f) Membrane intensity expression of epithelial syndecan-1 in a solid/ multicystic ameloblastoma. The intensity of stromal expression was considered a false-positive.

and Syn1E expression in ameloblastomas; a synchronous reduction in Ecad and Syn1E expression was found to be associated with a high expression of Syn1S. This is a potential factor contributing to the aggressive behavior of these tumors. Additional studies with more ameloblastoma cases are required and should include clinical follow-up and coexpression studies in which Ecad and Syn 1 are evaluated with proteins involved in cell proliferation, apoptosis, and growth factor proteins, as well as proteins involved in bone remodeling and ECM interactions. This can help to determine whether Syn1S expression and the simultaneous decreases in Ecad and Syn1E expression are also associated 
TABLE 4: Correlation between percentage expression and intensity of E-cadherin and syndecan-1 in UAM-L/I, UAM-M, and SMA.

\begin{tabular}{lccccc}
\hline Ameloblastoma & \% Ecad (SD)/intensity & Spearman $(\rho)$ & \% Syn1E (SD)/intensity & Spearman $(\rho)$ & \% Syn1S $($ SD) \\
\hline UAM-L/I & $65.5 \pm 32.6 / 2$ & $\rho=0.830$ & $64.7 \pm 24.8 / 2$ & $\rho=0.777$ & $36.4 \pm 24.9$ \\
UAM-M & $58 \pm 23.6 / 2$ & $\rho=0.655$ & $58.9 \pm 22.1 / 2$ & $\rho=0.744$ & $49.1 \pm 19$ \\
SMA & $49 \pm 28 / 2$ & $\rho=0.573$ & $49.5 \pm 27.1 / 2$ & $\rho=0.838$ & $53.9 \pm 32.6$ \\
*Significance & & $p=0.001$ & & $p=0.001$ \\
\hline
\end{tabular}

${ }^{*}$ Statistical significance at $p<0.05$, expression versus intensity. Ecad = E-cadherin expression; Syn1E = epithelial expression of syndecan-1; Syn1S = stromal expression of syndecan-1.

with mechanisms of bone remodeling, tumor invasion, and cellular immortality.

\section{Conflicts of Interest}

The authors declare that they have no conflicts of interest.

\section{References}

[1] G. B. Giraddi, K. Arora, and A. M. Saifi, "Ameloblastoma: a retrospective analysis of 31 cases," Journal of Oral Biology and Craniofacial Research, vol. 7, no. 3, pp. 206-211, 2017.

[2] A. K. El-Naggar, J. K. Chan, T. Takata, J. R. Grandis, and P. J. Slootweg, Eds., WHO Classification Head and Neck Tumours, IARC Press, Lyon, France, 4th edition, 2017.

[3] R. González-González, N. Molina-Frechero, P. Damian-Matsumura, and R. Bologna-Molina, "Molecular markers of cell adhesion in ameloblastomas. An update," Medicina Oral Patología Oral y Cirugia Bucal, vol. 19, no. 1, pp. e8-e14, 2014.

[4] K. Kurioka, M. Wato, T. Iseki, A. Tanaka, and S. Morita, "Differential expression of the epithelial mesenchymal transition factors Snail, Slug, Twist, TGF- $\beta$, and E-cadherin in ameloblastoma," Medical Molecular Morphology, vol. 50, no. 2, pp. 68-75, 2017.

[5] P. Balasundaram, M. Singh, A. Dinda, A. Thakar, and R. Yadav, "Study of $\beta$-catenin, E-cadherin and vimentin in oral squamous cell carcinoma with and without lymph node metastases," Diagnostic Pathology, vol. 9, no. 1, p. 145, 2014.

[6] Z. Chang, H. Zhou, and Y. Liu, "Promoter methylation and polymorphism of E-cadherin gene may confer a risk to prostate cancer: a meta-analysis based on 22 studies," Tumor Biology, vol. 35, no. 10, pp. 10503-10513, 2014.

[7] S.-L. Luo, Y.-G. Xie, Z. Li, J.-H. Ma, and X. Xu, "E-cadherin expression and prognosis of oral cancer: a meta-analysis," Tumour Biology, vol. 35, no. 6, pp. 5533-5537, 2014.

[8] A. Binder Gallimidi, G. Nussbaum, E. Hermano et al., "Syndecan-1 deficiency promotes tumor growth in a murine model of colitis-induced colon carcinoma," PLoS One, vol. 12, no. 3, article e0174343, 2017.

[9] A. Anttonen, M. Kajanti, P. Heikkilä, M. Jalkanen, and H. Joensuu, "Syndecan-1 expression has prognostic significance in head and neck carcinoma," British Journal of Cancer, vol. 79, no. 3-4, pp. 558-564, 1999.

[10] A. Matsumoto, M. Ono, Y. Fujimoto, R. L. Gallo, M. Bernfield, and Y. Kohgo, "Reduced expression of syndecan-1 in human hepatocellular carcinoma with high metastatic potential," International Journal of Cancer, vol. 74, no. 5, pp. 482491, 1997.

[11] J. O. Pulkkinen, M. Penttinen, M. Jalkanen, P. Klemi, and R. Grénman, "Syndecan-1: a new prognostic marker in laryngeal cancer," Acta Oto-Laryngologica, vol. 117, no. 2, pp. 312-315, 1997.

[12] R. Bologna-Molina, A. Mosqueda-Taylor, E. Lopez-Corella et al., "Syndecan-1 (CD138) and Ki-67 expression in different subtypes of ameloblastomas," Oral Oncology, vol. 44, no. 8, pp. 805-811, 2008.

[13] M. R. Akl, P. Nagpal, N. M. Ayoub et al., "Molecular and clinical profiles of syndecan-1 in solid and hematological cancer for prognosis and precision medicine," Oncotarget, vol. 6, no. 30, pp. 28693-28715, 2015.

[14] R. Bologna-Molina, R. González-González, A. Mosqueda-Taylor, N. Molina-Frechero, P. Damian-Matsumura, and H. Dominguez-Malagon, "Expression of syndecan-1 in papillary carcinoma of the thyroid with extracapsular invasion," Archives of Medical Research, vol. 41, no. 1, pp. 33-37, 2010.

[15] P. Leocata, D. Villari, C. Fazzari, M. Lentini, C. Fortunato, and P. A. Nicòtina, "Syndecan-1 and Wingless-type protein-1 in human ameloblastomas," Journal of Oral Pathology and Medicine, vol. 36, no. 7, pp. 394-399, 2007.

[16] O. Al-Otaibi, R. Khounganian, S. Anil, and R. Rajendran, "Syndecan-1 (CD 138) surface expression marks cell type and differentiation in ameloblastoma, keratocystic odontogenic tumor, and dentigerous cyst," Journal of Oral Pathology \& Medicine, vol. 42, no. 2, pp. 186-193, 2013.

[17] K. M. A. Pereira, B. A. do Amaral, B. R. M. dos Santos, H. C. Galvão, R. de Almeida Freitas, and L. B. de Souza, "Immunohistochemical expression of E-cadherin and $\beta$-catenin in ameloblastomas and tooth germs," Oral Surgery, Oral Medicine, Oral Pathology, Oral Radiology, and Endodontology, vol. 109, no. 3, pp. 425-431, 2010.

[18] H. Kumamoto and K. Ooya, "Expression of E-cadherin and alpha-catenin in epithelial odontogenic tumors: an immunohistochemical study," Journal of Oral Pathology and Medicine, vol. 28, no. 4, pp. 152-157, 1999.

[19] M. Martinez-Martinez, A. Mosqueda-Taylor, R. Carlos-Bregni et al., "Comparative histological and immunohistochemical study of ameloblastomas and ameloblastic carcinomas," Medicina Oral Patología Oral y Cirugia Bucal, vol. 22, no. 33, 2017.

[20] V. J. Tuominen, T. T. Tolonen, and J. Isola, "ImmunoMembrane: a publicly available web application for digital image analysis of HER2 immunohistochemistry," Histopathology, vol. 60, no. 5, pp. 758-767, 2012.

[21] J. J. V. Pinheiro, V. M. Freitas, A. I. S. Moretti, A. G. Jorge, and R. G. Jaeger, "Local invasiveness of ameloblastoma. Role played by matrix metalloproteinases and proliferative activity," Histopathology, vol. 45, no. 1, pp. 65-72, 2004.

[22] R. J. Cabay, "An overview of molecular and genetic alterations in selected benign odontogenic disorders," Archives of Pathology \& Laboratory Medicine, vol. 138, no. 6, pp. 754-758, 2014. 
[23] V. Nikolova, C. Y. Koo, S. A. Ibrahim et al., "Differential roles for membrane-bound and soluble syndecan-1 (CD138) in breast cancer progression," Carcinogenesis, vol. 30, no. 3, pp. 397-407, 2009.

[24] M. S. Filla, P. Dam, and A. C. Rapraeger, "The cell surface proteoglycan syndecan-1 mediates fibroblast growth factor-2 binding and activity," Journal of Cellular Physiology, vol. 174, no. 3, pp. 310-321, 1998.

[25] P. Mukunyadzi, K. Liu, E. Y. Hanna, J. Y. Suen, and C.-Y. Fan, "Induced expression of syndecan-1 in the stroma of head and neck squamous cell carcinoma," Modern Pathology, vol. 16, no. 8, pp. 796-801, 2003.

[26] M. Ohashi, T. Kusumi, F. Sato et al., "Expression of syndecan1 and E-cadherin is inversely correlated with poor patient's prognosis and recurrent status of extrahepatic bile duct carcinoma," Biomedical Research, vol. 30, no. 2, pp. 79-86, 2009.

[27] R. A. Safadi, B. F. Quda, and H. M. Hammad, "Immunohistochemical expression of K6, K8, K16, K17, K19, maspin, syndecan-1 (CD138), $\alpha$-SMA, and Ki-67 in ameloblastoma and ameloblastic carcinoma: diagnostic and prognostic correlations," Oral Surgery, Oral Medicine, Oral Pathology and Oral Radiology, vol. 121, no. 4, pp. 402-411, 2016.

[28] E. R. Fregnani, D. E. da Cruz Perez, O. P. de Almeida et al., "BRAF-V600E expression correlates with ameloblastoma aggressiveness," Histopathology, vol. 70, no. 3, pp. 473484, 2017.

[29] C. Jiang, Q. Zhang, R. M. Shanti et al., "Mesenchymal stromal cell-derived interleukin-6 promotes epithelial-mesenchymal transition and acquisition of epithelial stem-like cell properties in ameloblastoma epithelial cells," Stem Cells, vol. 35, no. 9, pp. 2083-2094, 2017.

[30] A. Kourtidis, R. Lu, L. J. Pence, and P. Z. Anastasiadis, “A central role for cadherin signaling in cancer," Experimental Cell Research, vol. 358, no. 1, pp. 78-85, 2017.

[31] K. Heikinheimo, K. J. Jee, T. Niini et al., "Gene expression profiling of ameloblastoma and human tooth germ by means of a cDNA microarray," Journal of Dental Research, vol. 81, no. 8, pp. 525-530, 2002.

[32] S. Saunders, M. Jalkanen, S. O'Farrell, and M. Bernfield, "Molecular cloning of syndecan, an integral membrane proteoglycan," The Journal of Cell Biology, vol. 108, no. 4, pp. 1547-1556, 1989.

[33] E. R. Fregnani, D. E. da Cruz Perez, O. P. de Almeida, L. P. Kowalski, F. A. Soares, and F. de Abreu Alves, "Clinicopathological study and treatment outcomes of 121 cases of ameloblastomas," International Journal of Oral and Maxillofacial Surgery, vol. 39, no. 2, pp. 145-149, 2010.

[34] A. Florescu, C. Mărgăritescu, C. E. Simionescu, and A. Stepan, "Immunohistochemical expression of MMP-9, TIMP-2, Ecadherin and vimentin in ameloblastomas and their implication in the local aggressive behavior of these tumors," Romanian Journal of Morphology and Embryology= Revue Roumaine de Morphologie et Embryologie, vol. 53, no. 4, pp. 975-984, 2012. 


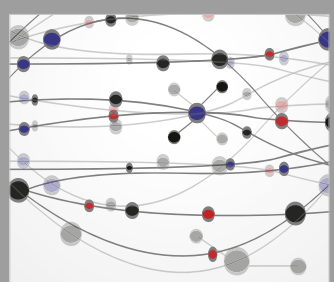

The Scientific World Journal
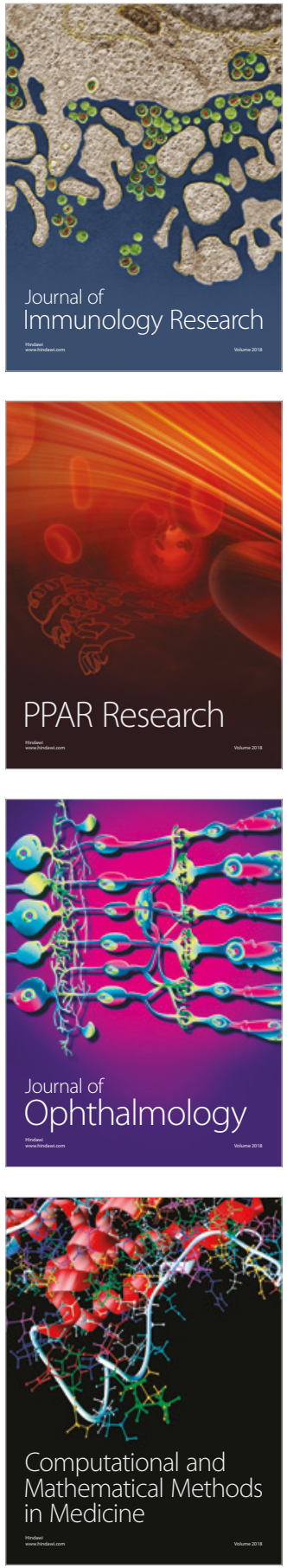

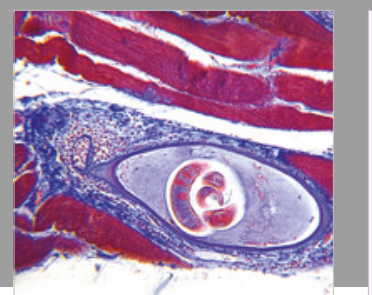

Gastroenterology Research and Practice

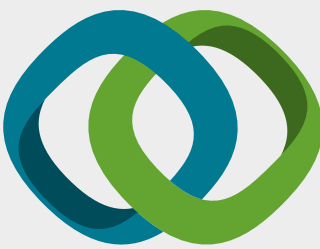

\section{Hindawi}

Submit your manuscripts at

www.hindawi.com
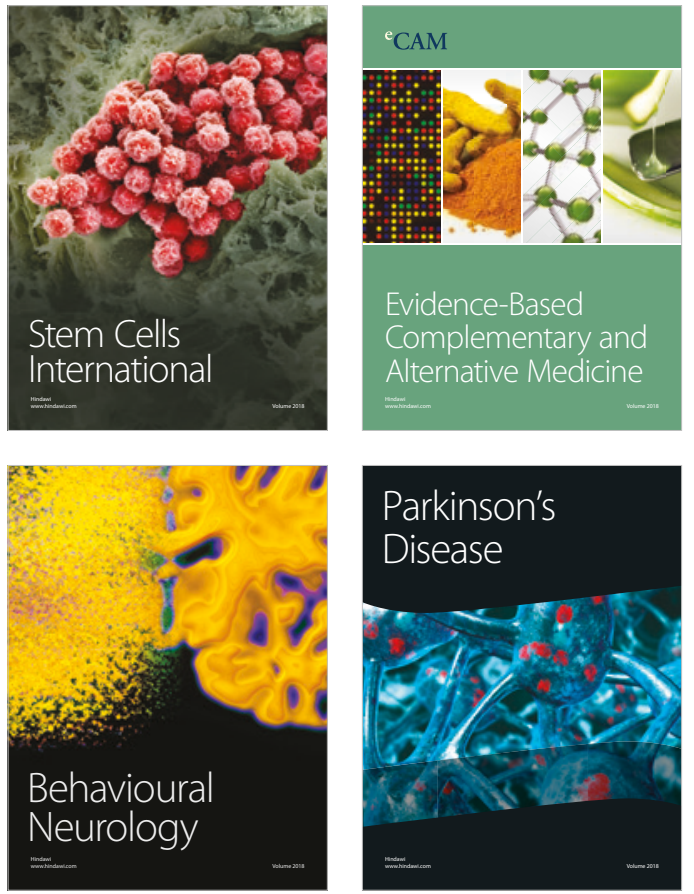

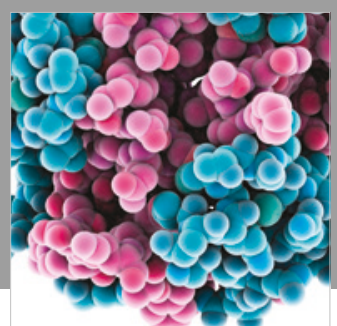

ournal of

Diabetes Research

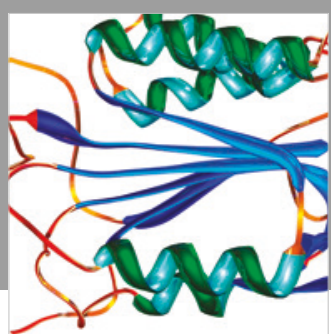

Disease Markers
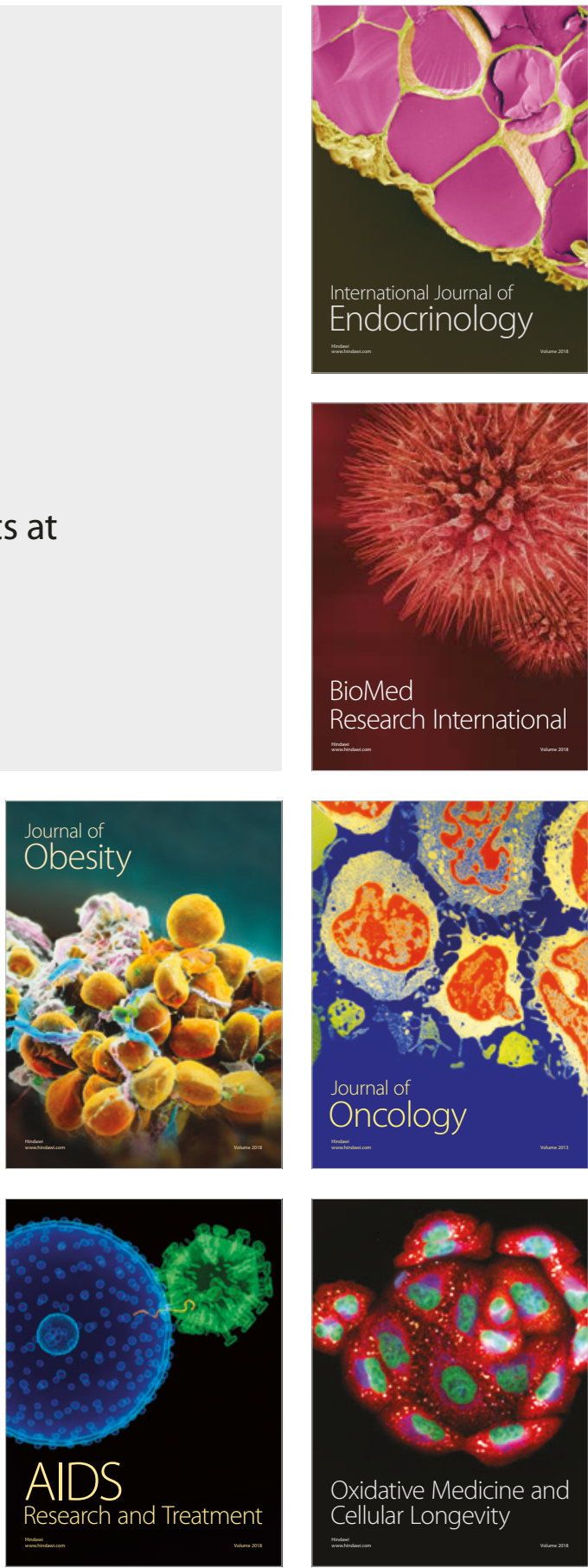